\title{
Rendimiento y composición mineral del forraje de una pradera permanente fertilizada con magnesio(1)
}

\author{
Oscar Balocchi( ${ }^{(2)}$, Dante Pinochet ${ }^{(2)}$, Fernando Wittwer ${ }^{(3)}$, Pedro Contreras $^{(3)}$, \\ Rodrigo Echeverria(2) y Francisco Guzmán ${ }^{(2)}$
}

\begin{abstract}
Resumen - Como una alternativa de manejo agronómico de la hipomagnesemia en rebaños a pastoreo se estudió, durante dos años, el efecto de la fertilización magnésica $\left(0,24,48\right.$ y $96 \mathrm{~kg} \mathrm{ha}^{-1}$ año-1 $\mathrm{de} \mathrm{Mg}$ ) sobre la concentración de magnesio, calcio, potasio y sodio y sus relaciones en la materia seca del forraje cosechado de una pradera permanente compuesta por Lolium perenne y Trifolium repens. El ensayo se realizó en un suelo Hapludand (Serie Valdivia) con un nivel inicial de $0,8 \mathrm{cmol}_{\mathrm{c}} \mathrm{kg}^{-1}$ de Mg. No se detectó un efecto de la fertilización magnésica sobre la producción de materia seca de la pradera, pero la concentración de magnesio en la materia seca se incrementó proporcionalmente a la dosis de magnesio aplicada. Considerando el promedio ponderado anual el aumento de la concentración de magnesio fue de 0,006 y $0,012 \mathrm{~g} \mathrm{~kg}^{-1}$ de materia seca por cada $\mathrm{kg}$ de magnesio aplicado, durante el primero y segundo año, respectivamente. La fertilización magnésica disminuyó la concentración de calcio, pero no afectó la concentración de potasio ni de sodio en la materia seca del forraje cosechado. Las relaciones entre $\mathrm{Ca} / \mathrm{Mg}, \mathrm{K} / \mathrm{Mg}$ y Na/Mg en general disminuyeron en la medida que aumentó la dosis de fertilización con magnesio.
\end{abstract}

Términos para índice: abonos, contenido de materia seca, manejo de praderas.

Yield and forage mineral composition of a permanent pasture fertilized with magnesium

Abstract - This research was conducted for two years to evaluate magnesium fertilizer application $(0$, 24, 48 and $96 \mathrm{~kg} \mathrm{ha}^{-1} \mathrm{year}^{-1}$ of $\mathrm{Mg}$ ) on a permanent pasture composed by Lolium perenne and Trifolium repens as an agronomic alternative of hypomagnesaemia management in grazing herds. The effect on magnesium, calcium, potassium and sodium concentration in the pasture dry matter and the ratio between cations were evaluated. The research was carried out on an Hapludand (Valdivia serie) with an initial available magnesium content of $0.8 \mathrm{cmol}_{\mathrm{c}} \mathrm{kg}^{-1}$. Pasture dry matter production was not affected by magnesium fertilization, nevertheless, the concentration of magnesium in the pasture dry matter increased proportionally to the magnesium applied. A weighed average showed an increase of 0.006 and $0.012 \mathrm{~g} \mathrm{~kg}^{-1}$ of dry matter for each $\mathrm{kg}$ of magnesium applied during the first and second year, respectively. In addition, the magnesium fertilization decreased the calcium dry matter concentration, but it did not affect potassium and sodium dry matter concentration. The ratios between calcium/magnesium, potassium/magnesium and sodium/magnesium decreased with the increase of the magnesium fertilization rate.

Index terms: fertilizers, dry matter content, grassland management.

\section{Introducción}

En la zona Sur de Chile la pradera permanente en pastoreo directo constituye el recurso más utilizado

(1) Aceptado para publicación en 30 de octubre de 2000 .

(2) Universidad Austral de Chile (UACh), Facultad de Ciencias Agrarias, Casilla 567 Valdivia, Chile. E-mail: obalocch@uach.cl, pinochet@uach.cl, seproani@uach.cl

(3) UACh, Facultad de Ciencias Veterinarias. E-mail: fwittwer@uach.cl,pcontrer@uach.cl en la alimentación del ganado bovino (Balocchi, 1999). En este recurso es deseable que se encuentren incorporados la mayor parte de los nutrientes que el animal requiere para su crecimiento y producción. Sin embargo, producto de una inadecuada concentración de $\mathrm{Mg}$ en el forraje y sus relaciones con la concentración de K, se genera con frecuencia, en rumiantes en pastoreo, la alteración metabólica hipomagnesemia o tetanía de los pastos, que afecta a rebaños con manejo intensivo y de pequeños productores de leche (Contreras et al., 1996). 
Según Wittwer et al. (1997), la hipomagnesemia afecta como una alteración metabólica de carácter subclínico a un porcentaje elevado de animales en un grupo mantenido en condiciones similares de manejo. En Chile, la prevalencia estimada es de 7\% y en la Décima Región es del 12\%, zona donde se encuentra la mayor concentración de la masa ganadera del país. Desde fines de otoño a inicios de primavera, el $38 \%$ de las vacas con decúbito de origen metabólico en el sur de Chile presentan hipomagnesemia, con una mayor ocurrencia a fines de gestación y en las primeras semanas de lactancia (Wittwer et al., 1993). Estos antecedentes demuestran la importancia de definir medidas de prevención para evitar las pérdidas económicas que provoca. Con este propósito, Wittwer et al. (1995) demostraron la eficacia de la administración oral a los animales de óxido de $\mathrm{Mg}$ y sulfato de $\mathrm{Mg}$ para recuperar los valores sanguíneos de $\mathrm{Mg}$ en vacas con hipomagnesemia inducida mediante ayuno. Sin embargo, el control de la hipomagnesemía en animales en pastoreo se hace particularmente difícil debido a lo repentino de la presentación de esta afección y a la dificultad de mantener una ingesta adecuada de $\mathrm{Mg}$ por medio de un sistema eficiente $\mathrm{y}$ práctico de suplementación.

En el desarrollo de los sistemas ganaderos se utiliza cada vez más a la pradera como fuente base de alimento, reduciendo la dependencia de concentrados. Esto transforma, en muchos casos, a la pradera en la única fuente nutricional haciendo que el balance de $\mathrm{Mg}$ del animal sea dependiente del contenido y disponibilidad de los minerales en el forraje consumido (Moseley \& Baker, 1991). Además, recientemente se ha enfatizado la necesidad de administrar el $\mathrm{Mg}$ en forma continua y en la cantidad requerida en el período de riesgo. El problema de agregar sales de $\mathrm{Mg}$ es que estas alteran la palatabilidad de la ración (Wittwer et al., 1997). En adición, el exceso en el consumo de Mg no solo constituye un gasto innecesario, ya que no ayuda a formar reservas orgánicas, sino que incluso puede llegar a ser peligroso, ya que compite con la absorción de Ca y P (Payne, 1981).

Las estrategias de suplementación magnésica son más factibles de utilizar en vacas de lechería debido al acceso directo a los animales, en los momentos de ordeña. En el ganado de carne la suplementación con $\mathrm{Mg}$ ha sido efectiva en reducir la incidencia de casos clínicos o muertes por tetania hipomagnesémica (Wittwer et al., 1997). Sin embargo, en ganado alimentado exclusivamente en base a pastoreo de praderas se dificulta su prevención, dado que se requiere de un procedimiento que permita el consumo voluntario de las fuentes de $\mathrm{Mg}$ y se pierde el control de la cantidad ingerida por los animales (Wittwer et al., 1997).

La presente investigación tuvo el objetivo de determinar el efecto de la fertilización magnésica sobre la producción anual de materia seca de la pradera, el contenido de $\mathrm{Mg}$ del forraje a través del año y las concentraciones de $\mathrm{Ca}, \mathrm{K}$ y $\mathrm{Na}$ en la materia seca del forraje cosechado.

\section{Material y Métodos}

La investigación se realizó en el predio experimental Vista Alegre de la Universidad Austral de Chile, entre mayo de 1992 a abril de 1994. El sitio del ensayo corresponde a un suelo serie Valdivia (Typic Hapludand), de topografía ligeramente ondulada y sin problemas de drenaje. El clima del sector se caracteriza por una temperatura promedio anual de $12,1^{\circ} \mathrm{C}$, con un valor medio mensual máximo de $16,9^{\circ} \mathrm{C}$ en enero y un mínimo de $7,6^{\circ} \mathrm{C}$ en julio. La precipitación promedio anual es de $2.500 \mathrm{~mm}$. Los valores de precipitación mensual durante el periodo experimental se presentan en el Cuadro 1.

Cuadro 1. Precipitación mensual ( $\mathrm{mm}$ ) durante el periodo experimental (mayo de 1992 a abril de 1994) y promedio mensual de 35 años en el agroecosistema de Valdivia.

\begin{tabular}{lrrc}
\hline Mes & Año 1 & Año 2 & $\begin{array}{c}\text { Promedio 35 } \\
\text { años }\end{array}$ \\
\hline Mayo & 392,7 & 464,2 & 369 \\
Junio & 408,0 & 501,9 & 408 \\
Julio & 209,6 & 489,1 & 380 \\
Agosto & 193,4 & 155,2 & 316 \\
Septiembre & 217,9 & 114,5 & 211 \\
Octubre & 273,4 & 133,1 & 125 \\
Noviembre & 71,7 & 61,5 & 128 \\
Diciembre & 148,3 & 211,8 & 110 \\
Enero & 41,2 & 15,6 & 64 \\
Febrero & 12,4 & 18,0 & 68 \\
Marzo & 247,7 & 28,6 & 107 \\
Abril & 402,5 & 200,3 & 220 \\
\hline Total & $2.372,8$ & $2.393,8$ & 2.506 \\
\hline
\end{tabular}


El ensayo fue establecido en un sitio que presentó un valor inicial de $\mathrm{Mg}$ intercambiable de $0,8 \mathrm{cmol}_{\mathrm{c}} \mathrm{kg}^{-1}$, ligeramente ácido y con una suma de bases de $5,1 \mathrm{cmol}_{\mathrm{c}} \mathrm{kg}^{-1}$. Sobre una pradera permanente mixta sembrada en marzo de 1992 se aplicaron los tratamientos de fertilización magnésica en cobertura. El fertilizante utilizado fue óxido de $\mathrm{Mg}$ y aplicado en abril de ambos años. La composición botánica de la pradera fue de Lolium perenne cv. Nui y Trifolium repens $\mathrm{cv}$. Huia.

Se utilizó un diseño de bloques completos al azar, con cuatro tratamientos y cuatro repeticiones. Los tratamientos correspondieron a $0,24,48$ y $96 \mathrm{~kg} \mathrm{ha}^{-1} \mathrm{año}^{-1} \mathrm{de} \mathrm{Mg}$. La fertilización basal en la siembra correspondió a $48 \mathrm{~kg} \mathrm{ha}^{-1}$ de $\mathrm{P}$ (como superfosfato triple), $50 \mathrm{~kg} \mathrm{ha}^{-1} \mathrm{de} \mathrm{K}$ (como muriato de $\mathrm{K}$ ), $54 \mathrm{~kg} \mathrm{ha}^{-1}$ de $\mathrm{N}$ y $6 \mathrm{~kg} \mathrm{ha}^{-1}$ de $\mathrm{Mg}$ (como nitromag). Adicionalmente, se aplicaron $45 \mathrm{~kg} \mathrm{ha}^{-1}$ de N, $96 \mathrm{~kg} \mathrm{ha}^{-1}$ de P y $198 \mathrm{~kg} \mathrm{ha}^{-1}$ de S (como superfosfato normal) en septiembre del primer año y en el segundo año $70 \mathrm{~kg} \mathrm{ha}^{-1}$ de $\mathrm{N}$ (como salitre sódico), $52 \mathrm{~kg} \mathrm{ha}^{-1}$ de $\mathrm{P}$ (como superfosfato triple), $50 \mathrm{~kg} \mathrm{ha}^{-1}$ de $\mathrm{K}$ y $12 \mathrm{~kg} \mathrm{ha}^{-1}$ de S (como sulfato de K) en agosto de 1993.

El forraje fue cosechado cada 30 días o cuando la pradera alcanzaba $20 \mathrm{~cm}$ de altura, simulando un manejo de pastoreo. Se dejó como altura de residuo $4 \mathrm{~cm}$. Los cortes fueron realizados mediante una segadora Quantum modelo Garden Pride 21". El forraje cosechado fue pesado en fresco y secado por 48 horas a $60^{\circ} \mathrm{C}$ en una estufa de aire forzado. El contenido de $\mathrm{Ca}, \mathrm{Mg}$, Nay K se determinó en submuestras del forraje seco, mediante calcinación y posterior determinación por espectrofotometría de absorción atómica (Rowell, 1996). En abril del año 1992 y 1994 se colectaron muestras de suelo de 0-20 cm de profundidad, para evaluar la variación en la disponibilidad de las bases de intercambio. Estas fueron determinadas por extracción en acetato de amonio $1 \mathrm{M}$, pH 7 y posterior determinación por espectrofotometría de absorción atómica (Rowell, 1996).

Los resultados fueron sometidos a un análisis de varianza y comparación entre medias con el test de Waller-Duncan, considerando un nivel de significancia de $\mathrm{P}<0,05$. Para estos análisis se utilizó el programa estadístico SAS (v 5.0).

\section{Resultados y Discusión}

Los promedios totales anuales de producción de materia seca en los distintos tratamientos de fertilización con $\mathrm{Mg}$ se presentan en el Cuadro 2. No se observó un efecto de la fertilización magnésica sobre el rendimiento de materia seca de la pradera durante los dos años de estudio. Los valores de producción obtenidos están dentro del rango esperado para las praderas permanentes del agrosistema de Valdivia, con un sistema de corte frecuente y para una fertilización nitrogenada media (Pinochet, 1999). La falta de respuesta a la fertilización con $\mathrm{Mg}$ está en concordancia con el nivel inicial de $\mathrm{Mg}$ intercambiable del suelo, el cual se encuentra no limitando la productividad de las praderas (Pinochet, 1989). Estos resultados son concordantes con los obtenidos por McNaught et al. (1968), Metson \& Saunders (1978) y Sierra (1992) en ensayos de fertilización magnésica de praderas mixtas bajo pastoreo. Dado que no existieron diferencias significativas entre los tratamientos de $\mathrm{Mg}$, en la Figura 1 se muestra la distribución mensual de la tasa promedio de crecimiento de la pradera con su respectiva desviación estándar, en los dos años de evaluación.

La curva de la tasa de crecimiento observada es similar a la descrita para las praderas permanentes de esta zona con un estrés hídrico estival. El crecimiento invernal durante el primer año fue de alrededor de $10 \mathrm{~kg} \mathrm{ha}^{-1}$ día $^{-1}$ de MS y fue diferente

Cuadro 2. Producción de materia seca anual de los tratamientos durante el periodo experimental ${ }^{(1)}$.

\begin{tabular}{ccc}
\hline $\begin{array}{c}\text { Tratamiento } \\
\left(\mathrm{kg} \mathrm{ha}^{-1} \mathrm{de} \mathrm{Mg}\right)\end{array}$ & $\begin{array}{c}\text { Año 1992/93 } \\
-------\left(\mathrm{kg} \mathrm{ha}^{-1} \mathrm{año}^{-1} \text { Año } 1993 / 94\right. \\
\mathrm{de})------\end{array}$ \\
\hline 0 & $10.995 \mathrm{a}$ & $8.852 \mathrm{a}$ \\
24 & $10.057 \mathrm{a}$ & $8.895 \mathrm{a}$ \\
48 & $10.534 \mathrm{a}$ & $8.882 \mathrm{a}$ \\
96 & $11.086 \mathrm{a}$ & $8.686 \mathrm{a}$ \\
\hline
\end{tabular}

(1)Promedios de igual letra en la columna son estadísticamente iguales $(\mathrm{p}>0,05)$.

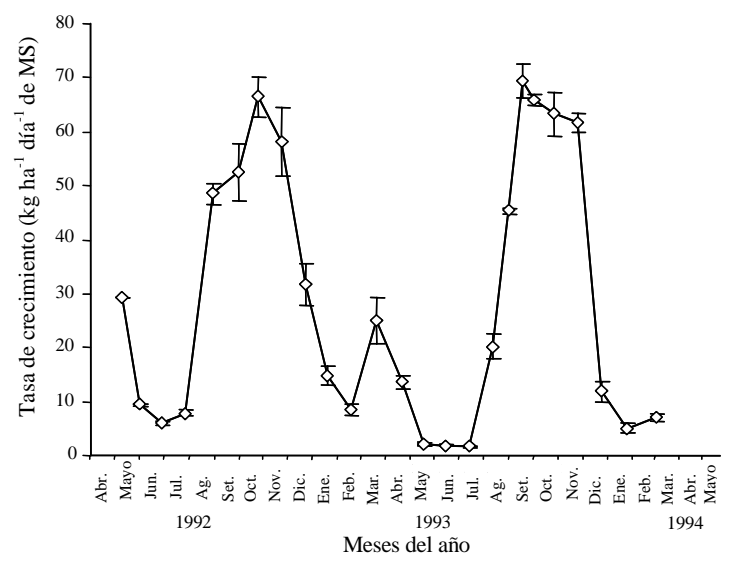

Figura 1. Distribución mensual de la tasa promedio de crecimiento de la pradera, durante dos años de evaluación.

Pesq. agropec. bras., Brasília, v. 36, n. 10, p. 1309-1317, out. 2001 
del observado en el segundo año en que los valores fueron muy inferiores (aproximadamente $2 \mathrm{~kg} \mathrm{ha}^{-1}$ día $^{-1}$ de MS). Es posible que la frecuente desfoliación a que fue sometida la pradera haya afectado el crecimiento invernal en éste año, no permitiéndole una adecuada recuperación, o que haya existido un posible efecto negativo de la excesiva precipitación registrada ese año durante los meses invernales. Esta fue de 298 mm más que lo registrado en un año normal para los meses de mayo, junio y julio y de $495 \mathrm{~mm}$ más que lo registrado en el primer año de evaluación (Figura 1). Desafortunadamente, esta última hipótesis no pudo ser corroborada ya que no existen estudios nacionales que muestren el efecto del exceso de la precipitación sobre el crecimiento invernal. El crecimiento primaveral, en ambos años, comenzó en las primeras semanas de agosto, alcanzado un máximo de aproximadamente $70 \mathrm{~kg} \mathrm{ha}^{-1}$ día $^{-1}$ de MS hacia fines de octubre a primeros días de noviembre, en el primer año y hacia mediados de octubre, en el segundo año. Esta alta tasa de crecimiento fue mantenida hasta diciembre, desde donde decae bruscamente para alcanzar una baja tasa de crecimiento en verano, cuyo mínimo está en el mes de febrero. Después del estrés hídrico estival y producto del incremento de las precipitaciones durante los meses de marzo, abril y mayo se observó un nuevo incremento en la tasa de crecimiento en el año 1993. Este incremento alcanzó un valor de $28 \mathrm{~kg} \mathrm{ha}^{-1}$ día $^{-1}$ de MS en el mes de abril. La menor tasa observada en otoño de 1994 se debió a una prolongación del período de sequía estival (Cuadro 1).

En el Cuadro 3 se muestran los valores de concentración relativa de $\mathrm{Mg}$ en la materia seca a través de los dos años de duración del experimento. La fertilización con $\mathrm{Mg}$ aumentó significativamente el contenido de $\mathrm{Mg}$ en el forraje inmediatamente después de las aplicaciones durante el periodo de estudio (mayo de 1992 y mayo de 1993). Este efecto puede deberse a que en el primer corte posterior a la aplicación, quede un remanente de fertilizante adherido al follaje, el cual afectó el valor de concentración medido. Esto explicaría la más alta concentración observada en el corte del 20 de mayo de 1993 que llegó a valores de 7,3 $\mathrm{g} \mathrm{kg}^{-1}$ de $\mathrm{Mg}$ en la dosis de $96 \mathrm{~kg} \mathrm{ha}^{-1}$ de $\mathrm{Mg}$. Sin embargo, estos valo- res son de utilidad agronómica ya que representan el contenido de $\mathrm{Mg}$ del forraje que estaría disponible para consumo animal. La variación observada en la concentración de $\mathrm{Mg}$ entre el tratamiento sin $\mathrm{Mg}$ y los fertilizados, muestran un incremento proporcional a la dosis de $\mathrm{Mg}$ aplicada. El efecto de la primera aplicación realizada posterior al corte de homogenización mostró un incremento de 0,37, 0,77 y 0,93 para los tratamientos 24,48 y $96 \mathrm{~kg} \mathrm{ha}^{-1}$ de $\mathrm{Mg}$. Este efecto, durante el segundo año, medido 15 días posterior a la aplicación del fertilizante, produjo un aumento de 0,94, 2,07 y 4,27 $\mathrm{g} \mathrm{kg}^{-1}$ de MS, respectivamente. Los efectos observados en los meses posteriores al primer corte posterior a la

Cuadro 3. Promedio de la concentración de magnesio ( $\mathrm{g} \mathrm{kg}^{-1}$ de materia seca) en el forraje para cada tratamiento por corte y promedio ponderado anual, en dos años de evaluación ${ }^{(1)}$.

\begin{tabular}{|c|c|c|c|c|}
\hline \multirow{2}{*}{$\begin{array}{l}\text { Fecha de } \\
\text { corte }\end{array}$} & \multicolumn{4}{|c|}{ Tratamiento $\left(\mathrm{kg} \mathrm{ha}^{-1} \mathrm{año}^{-1}\right.$ de $\left.\mathrm{Mg}\right)$} \\
\hline & 0 & 24 & 48 & 96 \\
\hline & \multicolumn{4}{|c|}{ Año 1} \\
\hline $19 / 5 / 92$ & $1,60 \mathrm{a}$ & $1,60 \mathrm{a}$ & $1,60 \mathrm{a}$ & $1,60 \mathrm{a}$ \\
\hline $17 / 6 / 92$ & $2,70 \mathrm{~b}$ & $3,07 \mathrm{ab}$ & $3,47 \mathrm{a}$ & $3,63 \mathrm{a}$ \\
\hline $15 / 7 / 92$ & $2,00 \mathrm{~b}$ & $2,37 \mathrm{a}$ & $2,47 \mathrm{a}$ & $2,47 \mathrm{a}$ \\
\hline $17 / 8 / 92$ & $2,50 \mathrm{~b}$ & $2,87 \mathrm{a}$ & $2,90 \mathrm{a}$ & $2,93 \mathrm{a}$ \\
\hline $25 / 9 / 92$ & $3,27 \mathrm{a}$ & $3,27 \mathrm{a}$ & $3,67 \mathrm{a}$ & $3,60 \mathrm{a}$ \\
\hline $20 / 10 / 92$ & $3,47 \mathrm{a}$ & $3,33 \mathrm{a}$ & $3,57 \mathrm{a}$ & $4,00 \mathrm{a}$ \\
\hline $16 / 11 / 92$ & $2,83 b$ & $2,90 \mathrm{~b}$ & $2,93 b$ & $3,37 \mathrm{a}$ \\
\hline $19 / 12 / 92$ & $3,00 \mathrm{~b}$ & $3,03 b$ & $3,36 \mathrm{ab}$ & $3,64 \mathrm{a}$ \\
\hline $14 / 1 / 93$ & $3,59 b$ & $3,95 \mathrm{ab}$ & $4,20 \mathrm{a}$ & $4,14 \mathrm{ab}$ \\
\hline $15 / 2 / 93$ & $3,44 \mathrm{a}$ & $3,57 \mathrm{a}$ & $3,57 \mathrm{a}$ & $3,87 \mathrm{a}$ \\
\hline $15 / 3 / 93$ & $2,89 \mathrm{~b}$ & $3,02 b$ & $3,10 \mathrm{~b}$ & $3,43 a$ \\
\hline $20 / 4 / 93$ & $2,84 \mathrm{c}$ & $2,97 \mathrm{bc}$ & $3,57 \mathrm{ab}$ & $3,89 \mathrm{a}$ \\
\hline \multirow[t]{2}{*}{$\begin{array}{l}\text { Promedio } \\
\text { ponderado }\end{array}$} & $2,96 \mathrm{c}$ & $3,13 b c$ & $3,34 \mathrm{ab}$ & $3,54 \mathrm{a}$ \\
\hline & \multicolumn{4}{|c|}{ Año 2} \\
\hline $20 / 5 / 93$ & $3,00 \mathrm{~b}$ & $3,94 b$ & $5,07 \mathrm{ab}$ & $7,27 \mathrm{a}$ \\
\hline $17 / 6 / 93$ & $2,78 \mathrm{~d}$ & $3,31 \mathrm{c}$ & $3,61 b$ & $4,11 \mathrm{a}$ \\
\hline $15 / 7 / 93$ & $2,51 \mathrm{c}$ & $3,03 b$ & $3,27 b$ & $3,92 \mathrm{a}$ \\
\hline $16 / 8 / 93$ & $1,90 \mathrm{c}$ & $2,23 b c$ & $2,53 b$ & $2,95 \mathrm{a}$ \\
\hline $13 / 9 / 93$ & $1,94 \mathrm{~d}$ & $2,24 \mathrm{c}$ & $2,43 b$ & $2,76 a$ \\
\hline $30 / 9 / 93$ & $1,83 \mathrm{c}$ & $2,19 b$ & $2,45 \mathrm{ab}$ & $2,67 \mathrm{a}$ \\
\hline $14 / 10 / 93$ & $2,14 \mathrm{c}$ & $2,50 \mathrm{bc}$ & $2,86 a b$ & $2,92 \mathrm{a}$ \\
\hline $3 / 11 / 93$ & $2,19 \mathrm{c}$ & $2,56 \mathrm{~b}$ & $2,63 b$ & $2,98 \mathrm{a}$ \\
\hline $3 / 12 / 93$ & $2,37 \mathrm{~b}$ & $2,67 b$ & $2,65 b$ & $3,21 \mathrm{a}$ \\
\hline $3 / 1 / 94$ & $3,11 \mathrm{c}$ & $3,51 b c$ & $3,93 \mathrm{ab}$ & $4,32 \mathrm{a}$ \\
\hline $3 / 2 / 94$ & $3,15 \mathrm{a}$ & $2,98 \mathrm{a}$ & $3,38 \mathrm{a}$ & $3,33 \mathrm{a}$ \\
\hline $7 / 3 / 94$ & $2,69 \mathrm{a}$ & $2,52 \mathrm{a}$ & $2,77 \mathrm{a}$ & $2,78 \mathrm{a}$ \\
\hline $20 / 4 / 94$ & $2,47 \mathrm{~b}$ & $2,70 \mathrm{~b}$ & $2,85 \mathrm{~b}$ & $3,44 \mathrm{a}$ \\
\hline $\begin{array}{l}\text { Promedio } \\
\text { ponderado }\end{array}$ & $2,47 d$ & $2,80 \mathrm{c}$ & $3,11 b$ & $3,59 \mathrm{a}$ \\
\hline
\end{tabular}

Pesq. agropec. bras., Brasília, v. 36, n. 10, p. 1309-1317, out. 2001 
aplicación del fertilizante, se consideran como cambios en la concentración de $\mathrm{Mg}$ foliar producidos por la absorción de $\mathrm{Mg}$ del suelo. Durante los meses de invierno, de ambos años, la fertilización produjo un incremento significativo de la concentración de $\mathrm{Mg}$ en la materia seca de la pradera. Este incremento fue más pronunciado en el segundo año, en que las concentraciones varían entre $2,5 \mathrm{~g} \mathrm{~kg}^{-1}$ de MS en el tratamiento 0 hasta $3,9 \mathrm{~g} \mathrm{~kg}^{-1}$ de MS en el tratamiento $96 \mathrm{~kg} \mathrm{ha}^{-1} \mathrm{de} \mathrm{Mg}$. Este aumento pudo deberse al efecto acumulado de las dos aplicaciones de $\mathrm{Mg}$ al suelo y al efecto producido por el $\mathrm{Mg}$ aplicado en la fertilización basal, antes del establecimiento del ensayo.

Durante la primavera-verano, sin déficit hídrico evidente (septiembre, octubre, noviembre y diciembre) se mantuvo la diferencia entre los tratamientos fertilizados y de éstos comparados con el tratamiento sin fertilización. Al igual que lo observado en invierno el efecto fue significativamente mayor durante el segundo año. Durante los meses de verano afectados por el estrés hídrico (enero y febrero del año 1993 y enero, febrero y marzo del año 1994), no se detectaron diferencias significativas en la concentración de $\mathrm{Mg}$ en el forraje debido a los tratamientos. Parecería ser que el estrés hídrico afectó la absorción de $\mathrm{Mg}$ por las plantas desde el suelo. Finalmente en abril, cuando el estrés hídrico es superado, las diferencias en concentración de $\mathrm{Mg}$ en la materia seca debida a los tratamientos de fertilización vuelven a ser estadísticamente significativas.

Un análisis de la concentración promedio ponderada a través de la producción de todo el año muestra diferencias significativas para los tratamientos de fertilización, siendo estos estadísticamente diferentes para cada tratamiento en el segundo año. El aumento relativo por sobre la concentración de $\mathrm{Mg}$ del tratamiento $0 \mathrm{~kg} \mathrm{ha}^{-1}$ de $\mathrm{Mg}$ permite calcular que la concentración promedio aumenta en 0,006 y $0,012 \mathrm{~g} \mathrm{~kg}^{-1}$ de MS por cada $\mathrm{kg} \mathrm{ha}^{-1} \mathrm{año}^{-1}$ de Mg aplicado en el primero y segundo año, respectivamente (Cuadro 3).

En el Cuadro 4 se muestra el promedio ponderado anual de las concentraciones de $\mathrm{Ca}, \mathrm{K}$ y Na en el forraje cosechado en cada tratamiento de fertilización con $\mathrm{Mg}$.

Para el Ca se observa una disminución significativa de la concentración en la medida que aumenta la dosis de fertilización con $\mathrm{Mg}$, en el segundo año de evaluación. Analizados los datos mediante regresión se puede observar que en ambos años existió un efecto depresor de la fertilización con $\mathrm{Mg}$ sobre la concentración de $\mathrm{Ca}$ en el forraje (Año 1: $\mathrm{y}=-0,0063 \mathrm{x}+5,25 ; \quad \mathrm{r}^{2}=0,93$ y Año 2 : $\left.\mathrm{y}=-0,0087 \mathrm{x}+4,296 ; \mathrm{r}^{2}=0,99\right)$. Efectos depresores de la concentración de Ca por altas concentraciones de otros cationes han sido reportadas por Marschner (1995).

La Figura 2 muestra la evolución mensual de la concentración de $\mathrm{Ca}$ en el forraje cosechado durante

Cuadro 4. Efecto de la fertilización con $\mathrm{Mg}$ en el contenido de calcio, potasio, sodio, y en las relaciones $\mathrm{Ca} / \mathrm{Mg}, \mathrm{K} / \mathrm{Mg}$ y $\mathrm{Na} / \mathrm{Mg}$ del forraje cosechado, en dos años ${ }^{(1)}$

\begin{tabular}{|c|c|c|c|c|c|c|}
\hline \multirow{2}{*}{$\begin{array}{l}\text { Tratamiento } \\
\left(\mathrm{kg} \mathrm{ha}^{-1} \mathrm{Mg}\right)\end{array}$} & \multicolumn{3}{|c|}{ Año 1992/93 } & \multicolumn{3}{|c|}{ Año 1993/94 } \\
\hline & Calcio & Potasio & Sodio & Calcio & Potasio & Sodio \\
\hline & \multicolumn{6}{|c|}{ (g de nutriente por kg de materia seca) } \\
\hline 0 & $5,18 \mathrm{a}$ & $36,7 \mathrm{a}$ & $2,59 \mathrm{a}$ & $4,27 \mathrm{a}$ & $29,2 \mathrm{~A}$ & $3,28 \mathrm{a}$ \\
\hline 24 & $5,14 \mathrm{a}$ & $33,4 b$ & $2,61 \mathrm{a}$ & $4,13 \mathrm{a}$ & $28,0 \mathrm{~A}$ & $3,18 \mathrm{a}$ \\
\hline 48 & $5,03 \mathrm{a}$ & $34,2 b$ & $2,55 \mathrm{a}$ & $3,87 \mathrm{ab}$ & $28,7 \mathrm{~A}$ & $3,03 \mathrm{a}$ \\
\hline \multirow[t]{3}{*}{96} & $4,60 \mathrm{a}$ & $36,3 \mathrm{a}$ & $2,66 \mathrm{a}$ & $3,46 \mathrm{~b}$ & $28,5 \mathrm{~A}$ & $2,86 \mathrm{a}$ \\
\hline & \multicolumn{6}{|c|}{ Relaciones entre nutrientes } \\
\hline & $\mathrm{Ca} / \mathrm{Mg}$ & $\mathrm{K} / \mathrm{Mg}$ & $\mathrm{Na} / \mathrm{Mg}$ & $\mathrm{Ca} / \mathrm{Mg}$ & $\mathrm{K} / \mathrm{Mg}$ & $\mathrm{Na} / \mathrm{Mg}$ \\
\hline 0 & 1,75 & 12,4 & 0,88 & 1,73 & 11,8 & 1,33 \\
\hline 24 & 1,64 & 10,7 & 0,83 & 1,48 & 10,0 & 1,14 \\
\hline 48 & 1,51 & 10,2 & 0,76 & 1,24 & 9,2 & 0,97 \\
\hline 96 & 1,30 & 10,3 & 0,75 & 0,96 & 7,9 & 0,80 \\
\hline
\end{tabular}

(1)Promedios de igual letra en la columna son estadísticamente iguales $(\mathrm{p}>0,05)$.

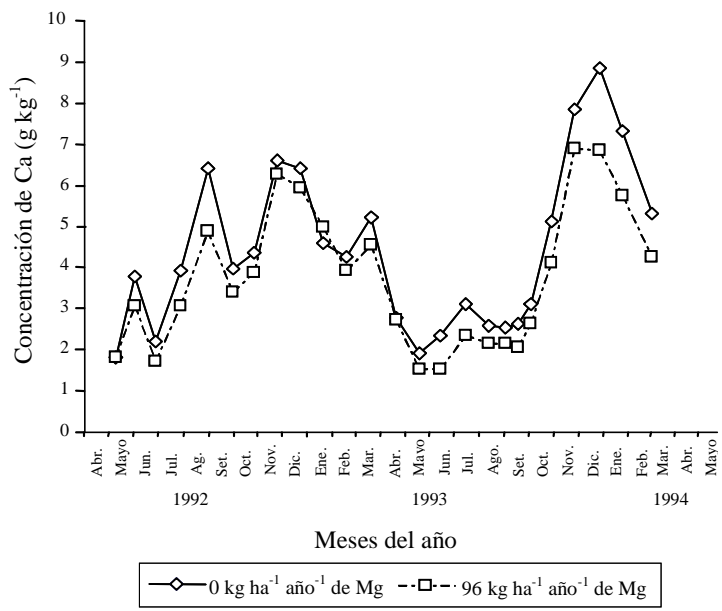

Figura 2. Evolución mensual de la concentración de calcio en el forraje durante dos años para el tratamiento sin fertilización con $\mathrm{Mg}$ y el tratamiento $96 \mathrm{~kg} \mathrm{ha}^{-1} \mathrm{año}^{-1}$ de Mg. 
dos años para el tratamiento sin fertilización con $\mathrm{Mg}$ y para el tratamiento $96 \mathrm{~kg} \mathrm{ha}^{-1} \mathrm{año}^{-1} \mathrm{de} \mathrm{Mg}$.

Se observa que la concentración de $\mathrm{Ca}$ en el tratamiento con fertilización con $\mathrm{Mg}$ disminuyó con respecto al control sin fertilización con $\mathrm{Mg}$, aunque la tendencia en variación estacional fue similar. Los mayores valores de $\mathrm{Ca}$ en el forraje en ambos años fue en los meses de noviembre, diciembre y enero. Este patrón de distribución estacional difiere de lo observado por Cuevas et al. (1983) y Anrique (1995), en que la concentración de Ca alcanzó un mínimo en estos meses con valores de $2 \mathrm{~g} \mathrm{~kg}^{-1}$ de MS. Esta diferencia podría ser explicada, al menos parcialmente, por la diferente composición botánica de las praderas evaluadas. En este estudio el porcentaje de leguminosas medido en el mes de noviembre fue en promedio de los tratamientos de $25,1 \%$ (Echeverría, 1995; Guzmán, 1996). Este porcentaje de leguminosa es más alto que el usualmente encontrado en las praderas permanentes del sur de Chile, que es menor al $10 \%$ para esta misma época (Balocchi \& Olivares, 1992). Los menores valores de concentración de Ca se presentaron en mayo junto al rebrotre otoñal. En este caso los valores son coincidentes con los reportados por Cuevas et al. (1983) y Anrique (1995). Por otra parte, la distribución anual de este elemento reportada por Metson \& Saunders (1978) coinciden con los niveles extremos observados en este estudio, con mínimos en invierno y máximos en verano. Sin embargo, no existe cosenso respecto a las variaciones estacionales del $\mathrm{Ca}$. En este sentido, las investigaciones de otros autores señalan cambios poco consistentes y evoluciones fluctuantes, no existiendo un modelo que caracterice su comportamiento anual (Spedding \& Diekmahns, 1972).

La concentración de $\mathrm{K}$ en el forraje tendió a mantenerse constante en los diferentes niveles de fertilización con $\mathrm{Mg}$ en ambos años, a pesar que variaciones significativas fueron detectadas para el primer año, pero que no mostraron una tendencia definida y no se mantuvieron en el segundo año. La concentración de $\mathrm{K}$ es alrededor de diez veces superior a la concentración de $\mathrm{Mg}$, por lo cual parece poco probable que la fertilización magnésica pueda afectar significativamente el contenido de K.
La Figura 3 muestra la concentración promedio de K y su desviación estándar observada durante los dos años de evaluación. Los valores de $\mathrm{K}$ de 35,2 y $28,6 \mathrm{~g} \mathrm{~kg}^{-1}$ de MS son mayores a los observados por Cuevas et al. (1983) para praderas permanentes de la zona sur con valores cercanos a $10 \mathrm{~g} \mathrm{~kg}^{-1}$ de MS. Esta diferencia podría estar explicada por el alto nivel de K intercambiable (Cuadro 5) al inicio del ensayo y a la fertilización potásica utilizada. Los mayores valores de $\mathrm{K}$ se presentan en mayo y septiembre en el primer y segundo año respectivamente, disminuyendo hasta alcanzar un mínimo durante el mes de enero de ambos años. Esta tendencia estacional es similar a la reportada por Cuevas et al. (1983) y estaría explicada por la ontogenia de las especies constituyentes de la pradera. Además se observó una declinación general en el contenido de $\mathrm{K}$ del forraje cosechado desde el inicio al final del

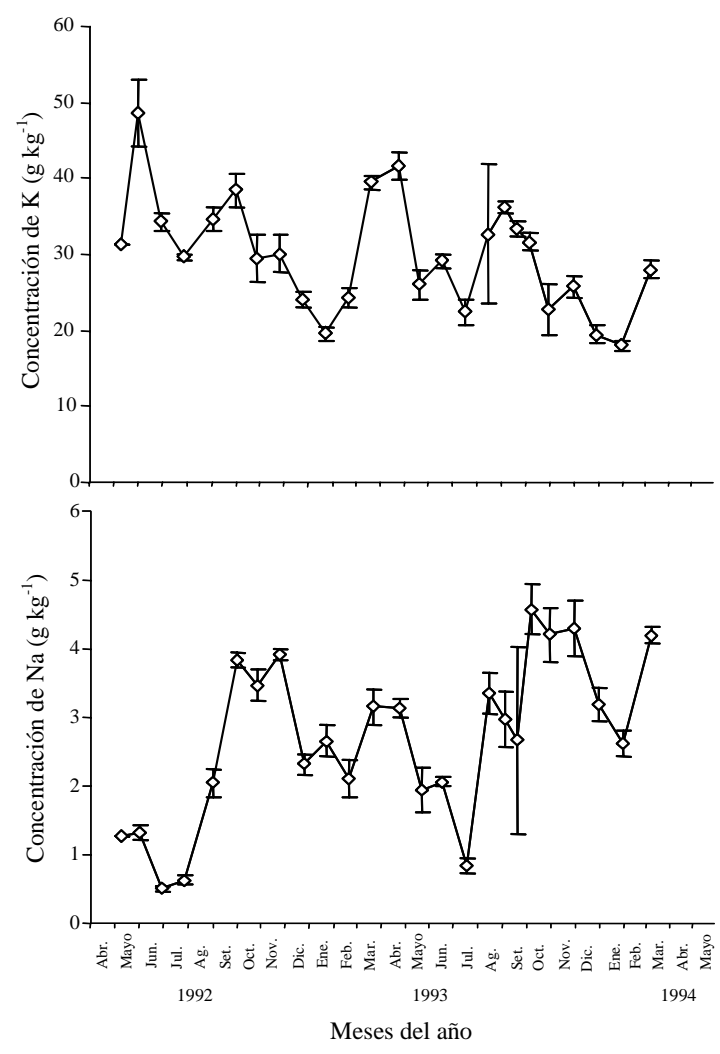

Figura 3. Evolución mensual de la concentración de potasio y de sodio en el forraje durante dos años para el promedio de los tratamientos. 
ensayo. Esta disminución es coincidente con una declinación en el contenido de $\mathrm{K}$ intercambiable del suelo a pesar de la fertilización potásica aplicada, indicando que esta fue insuficiente para mantener el nivel de K del suelo, dada la alta extracción de este nutriente en el forraje cosechado.

La concentración de $\mathrm{Na}$ en el forraje no fue afectada por los niveles de fertilización con $\mathrm{Mg}$, en ambos años de evaluación (Cuadro 4). La Figura 4 muestra la evolución de la concentración de $\mathrm{Na}$ en el forraje a través del periodo de evaluación. Al observar la distribución estacional de ambos años de evaluación, se evidencia un aumento de la concentración de este elemento en el forraje a partir del inicio de primavera, alcanzando valores máximos en esta estación. Los valores mínimos en ambos períodos de estudio se presentan en el mes de julio. Esta tendencia difiere a lo observado por McNaught et al. (1968) y Metson \& Saunders (1978) quienes indican que el Na no seguiría una tendencia estacional clara en gramíneas y leguminosas ya que sus niveles fluctuarían en forma aleatoria. La tendencia general, durante los dos años de evaluación, fue de un incremento del contenido promedio de $\mathrm{Na}$ en el forraje, lo que pudo haber sido causado por la fertilización nitrogenada con nitrato de Na y evidenciado, además, en el aumento del contenido de $\mathrm{Na}$ intercambiable en el suelo (Cuadro 5).

Las relaciones promedio anual entre $\mathrm{Ca}, \mathrm{K}$ y $\mathrm{Na}$ con respecto a $\mathrm{Mg}$ (Cuadro 4), muestran un decrecimiento en la medida que aumenta la fertilización con $\mathrm{Mg}$. La relación $\mathrm{Ca} / \mathrm{Mg}$ varía desde

Cuadro 5. Contenido de nutrientes disponibles en el suelo al inicio y término del ensayo.

\begin{tabular}{|c|c|c|}
\hline Parámetro & $\begin{array}{c}\text { Abril } 1992 \\
\text { (inicio) }\end{array}$ & $\begin{array}{c}\text { Abril } 1994 \\
\text { (término) }\end{array}$ \\
\hline $\mathrm{pH}$ (agua $1: 2,5)$ & 5,8 & 5,8 \\
\hline Materia orgánica $(\%)$ & 11,1 & 9,7 \\
\hline $\mathrm{N}$ mineral $\left(\mathrm{mg} \mathrm{kg}^{-1}\right)$ & 9,6 & 12,6 \\
\hline $\mathrm{P}$ aprovechable $\left(\mathrm{mg} \mathrm{kg}^{-1}\right)$ & 4,0 & 12,2 \\
\hline $\mathrm{K}$ intercambiable $\left(\mathrm{mg} \mathrm{kg}^{-1}\right)$ & 352,0 & 156,0 \\
\hline $\mathrm{Na}$ intercambiable $\left(\mathrm{cmol}_{\mathrm{c}} \mathrm{kg}^{-1}\right)$ & 0,23 & 0,31 \\
\hline $\mathrm{Ca}$ intercambiable $\left(\mathrm{cmol}_{\mathrm{c}} \mathrm{kg}^{-1}\right)$ & 3,20 & 3,33 \\
\hline \multicolumn{3}{|l|}{$\mathrm{Mg}$ intercambiable $\left(\mathrm{cmol}_{\mathrm{c}} \mathrm{kg}^{-1}\right)$} \\
\hline $0 \mathrm{~kg} \mathrm{ha}^{-1}$ de $\mathrm{Mg}$ & 0,80 & $0,62 \pm 0,08$ \\
\hline $24 \mathrm{~kg} \mathrm{ha}^{-1} \mathrm{de} \mathrm{Mg}$ & & $0,83 \pm 0,19$ \\
\hline $48 \mathrm{~kg} \mathrm{ha}^{-1}$ de $\mathrm{Mg}$ & & $1,05 \pm 0,11$ \\
\hline $96 \mathrm{~kg} \mathrm{ha}^{-1} \mathrm{de} \mathrm{Mg}$ & & $1,47 \pm 0,34$ \\
\hline
\end{tabular}

1,75 a 0,96 durante los dos años de estudio, dependiendo de la dosis de $\mathrm{Mg}$ aplicada. La relación $\mathrm{Na} / \mathrm{Mg}$ varió de 1,33 a 0,75 dependiendo del nivel de fertilización con $\mathrm{Mg}$ y de la dosis de $\mathrm{Na}$ aplicada. De acuerdo a estos resultados existe la posibilidad de modificar, al menos parcialmente, las relaciones entre los cationes a través de la fertilización, dependiendo del contenido del nutriente en el suelo. Dentro de las relaciones entre cationes la relación $\mathrm{K} / \mathrm{Mg}$ es determinante en el riesgo de ocurrencia de la hipomagnesemía (Sykes, 1993; Wittwer, 1996). La Figura 4 muestra la variación observada, a través del periodo de evaluación de la relación $\mathrm{K} / \mathrm{Mg}$ para los tratamientos 0 y $96 \mathrm{~kg} \mathrm{ha}^{-1} \mathrm{de} \mathrm{Mg}$. La tendencia general a través de los dos años es similar, mostrando una variación estacional la cual alcanza los mayores valores en los meses de otoño y primavera. Los valores más bajos de la relación se observaron en los meses de verano. La fertilización con $\mathrm{Mg}$ afectó la relación, alcanzando una menor relación $\mathrm{K} / \mathrm{Mg}$ en los tratamientos que recibieron fertilización con $\mathrm{Mg}$. Esta variación sugiere la posibilidad de manejo agronómico de esta relación, cuando los contenidos de $\mathrm{K}$ son altos disminuyendo el riesgo potencial de hipomagnesemía.

El nivel de $\mathrm{Mg}$ intercambiable residual varió con la fertilización con $\mathrm{Mg}$ (Cuadro 5). Existió una disminución del contenido de $\mathrm{Mg}$ cuando no se aplicó fertilización con $\mathrm{Mg}$ de $0,18 \mathrm{cmol}_{\mathrm{c}} \mathrm{kg}^{-1}$, después de

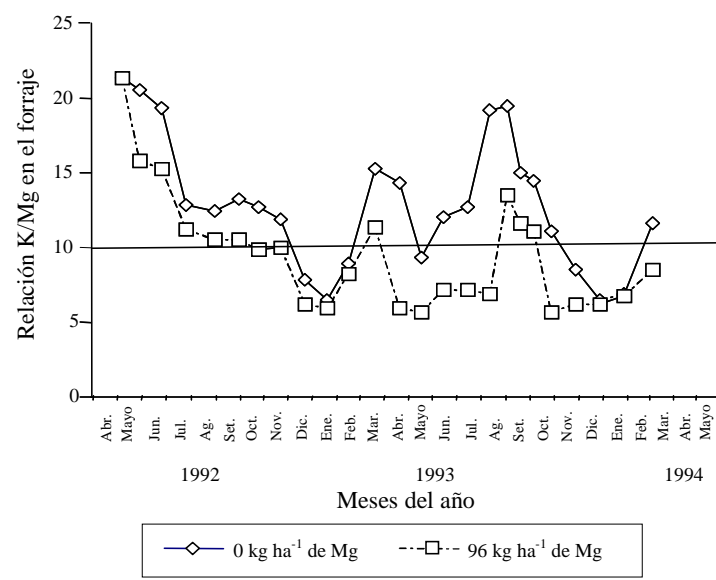

Figura 4. Variación a través del período de evaluación de la relación $\mathrm{K} / \mathrm{Mg}$ para los tratamientos 0 y $96 \mathrm{~kg} \mathrm{ha}^{-1}$ año ${ }^{-1} \mathrm{de} \mathrm{Mg}$. 
dos años de extracción. Esta disminución fue compensada con la aplicación de $24 \mathrm{~kg} \mathrm{ha}^{-1} \mathrm{de} \mathrm{Mg}$, sugiriendo que para este manejo correspondería a la fertilización de mantención. Con niveles superiores (48 y $96 \mathrm{~kg} \mathrm{ha}^{-1} \mathrm{de} \mathrm{Mg}$ ) se produce un aumento en el contenido de $\mathrm{Mg}$ intercambiable en el suelo, proporcional a la dosis aplicada. Las dosis de fertilización con $\mathrm{Mg}$ utilizadas no afectaron el $\mathrm{pH}$ del suelo, probablemente debido a que estas dosis serían insuficientes para modificar en forma significativa la concentración de protones del suelo.

\section{Conclusiones}

1. En un suelo con un nivel inicial de $0,8 \mathrm{cmol}_{\mathrm{c}} \mathrm{kg}^{-1} \mathrm{de} \mathrm{Mg}$, la fertilización magnésica no afecta significativamente la producción y distribución estacional de materia seca de una pradera permanente.

2. La fertilización con Mg afecta negativamente el contenido de $\mathrm{Ca}$ del forraje, con un decrecimiento proporcional a la dosis de $\mathrm{Mg}$ aplicada.

3. El contenido de $\mathrm{K}$ y Na del forraje no es afectado por la fertilización con Mg: las variaciones en el contenido de $\mathrm{K}$ y Na en el forraje son consistentes con sus variaciones en el contenido de estos nutrientes en el suelo.

4. Las relaciones promedio anual entre $\mathrm{Ca}, \mathrm{K} \mathrm{y}$ $\mathrm{Na}$ con respecto al $\mathrm{Mg}$, disminuyen en la medida que aumenta la dosis de fertilización con Mg.

\section{Agradecimientos}

Al Proyecto FONDECYT 92-0169 "Estudio sobre el diagnóstico, control y prevención de la hipomagnesemia bovina" por el financiamiento otorgado.

\section{Referencias}

ANRIQUE, R. Composición de alimentos para el ganado de la zona sur. Valdivia: Universidad Austral de Chile, 1995. $57 \mathrm{p}$.

BALOCCHI, O. Recursos forrajeros utilizados en producción de leche. In: LATRILLE, L. (Ed.). Producción animal 1999. Valdivia: Universidad Austral de Chile, 1999. p. 186-214. (Serie, B-22)
BALOCCHI, O.; OLIVARES, J. Leguminosas en praderas permanentes. In: LATRILLE, L.; BALOCCHI, O. (Ed.). Producción animal 1992. Valdivia: Universidad Austral de Chile, 1992. p. 33-58. (Serie, B-16).

CONTRERAS, P.; VALENZUELA, L.; WITTWER, F.; BÖHMWALD, H. Desbalances metabólicos nutricionales más frecuentes en rebaños de pequeños productores de leche, Valdivia, Chile. Archivos de Medicina Veterinaria, Valdivia, v. 28, n. 1, p. 39-50, 1996.

CUEVAS, E.; BALOCCHI, O.; ANRIQUE, R.; JORQUERA, M. Valor nutritivo de las principales especies de una pradera permanente en la Décima Región: II. Contenido de minerales. Agro Sur, Valdivia, v. 11, n. 1, p. 13-22, 1983.

ECHEVERRÍA, R. Efecto de la fertilización magnésica sobre el rendimiento y nivel de magnesio en una pradera permanente de la Décima Región. Valdivia: Universidad Austral de Chile, 1995. 53 p. Tesis Licenciado en Agronomía.

GUZMÁN, F. Efecto de la fertilización magnésica, en un segundo año de aplicación, sobre el rendimiento y nivel de magnesio en una pradera permanente de la Décima Región. Valdivia: Universidad Austral de Chile, 1996. 64 p. Tesis Licenciado en Agronomía.

McNAUGHT, K.; DOROFAEFF, F.; KARLOVSKY, J Effect of magnesium fertilizers and season on level of inorganic nutrients in a pasture on Hamilton clay loam. New Zealand Journal of Agricultural Research, New Zealand, v. 11, n. 3, p. 533-550, 1968.

MARSCHNER, H. Mineral nutrition of higher plants. 2. ed. San Diego, California: Academic, 1995. 889 p.

METSON, A.; SAUNDERS, W. Seasonal variations in chemical composition of pasture. I. Calcium, magnesium, potassium, sodium and phosphorus. New Zealand Journal of Agricultural Research, Wellington, v. 21, n. 2, p. 341353, 1978.

MOSELEY, G.; BAKER, D. The efficacy of a high magnesium grass cultivar in controlling hypomagnesemia in grazing animals. Grass and Forage Science, Oxford, v. 46 , n. 4 , p. 375-380, 1991.

PAYNE, J. Enfermedades metabólicas de los animales zootécnicos. Zaragoza: Acribia, 1981. 219 p.

PINOCHET, D. Estado nutricional de los suelos agrícolas de la zona sur. Próxima Década, Santiago, v. 77, n. 7, p. 1416,1989 
PINOCHET, D. Potencial productivo de las praderas permanentes de las Regiones IX y X. In: LATRILLE, L. (Ed.). Producción animal 1999. Valdivia: Universidad Austral de Chile,1999. p. 237-273. (Serie, B-22).

ROWELL, D. Soil science: methods and applications. Essex: A. W. Longman, 1996. 350 p.

SIERRA, C. Limitaciones edáficas para el crecimiento de leguminosas. In: LATRILLE, L.; BALOCCHI, O. (Ed.). Producción animal 1992. Valdivia: Universidad Austral de Chile, 1992. p. 1-15. (Serie, B-16).

SPEDDING C.; DIEKMAHNS, E. Grasses and legumes in British agriculture. Oxford: Alden and Mowbray, 1972.511 p.

SYKES, A. Hipomagnesemia en bovinos. In: JORNADAS CHILENAS DE BUIATRIA, 1., 1993, Osorno. Anales.. Osorno: Sociedad Chilena de Buiatria, 1993. p. 21-28.

WITTWER, F. Bases para el manejo de la hipomagnesemia bovina en el sur de Chile. In: LATRILLE, L. (Ed.).
Producción animal 1996. Valdivia: Universidad Austral de Chile, 1996. p. 119-130. (Serie, B-19).

WITTWER, F.; CONTRERAS, P.; KLEIN, R.; BÖHMWALD, H. Efecto de la administración de óxido de magnesio y sulfato de magnesio en vacas con hipomagnesemia inducida. Archivos de Medicina Veterinaria, Valdivia, v. 27, p. 19-28, 1995.

WITTWER, F.; CONTRERAS, P.; SILVA, N.; BÖHMWALD, H. Efecto de la suplementación con magnesio en el alimento y agua sobre el control de tetania hipomagnesémica en rebaños Hereford. Archivos de Medicina Veterinaria, Valdivia, v. 29, p. 25-33, 1997.

WITTWER, F.; HEUER, G.; CONTRERAS, P.; BÖHMWALD, H. Valores bioquímicos clínicos sangüíneos de vacas cursando con decúbito en el sur de Chile. Archivos de Medicina Veterinaria, v. 25, p. 83-88, 1993. 